\title{
Lévy Statistics and the Glassy Behavior of Light in Random Fiber Lasers
}

\author{
Cid B. de Araújo ${ }^{1, *}$, Anderson S. L. Gomes ${ }^{1}$ and Ernesto P. Raposo ${ }^{2}$ \\ 1 Departamento de Física, Universidade Federal de Pernambuco, Recife-PE 50670-901, Brazil; \\ anderson@df.ufpe.br \\ 2 Laboratório de Física Teórica e Computacional, Departamento de Física, Universidade Federal de \\ Pernambuco, Recife-PE 50670-901, Brazil; ernesto@df.ufpe.br \\ * Correspondence: cid@df.ufpe.br; Tel.: +55-81-2126-7630
}

Academic Editor: Boris Malomed

Received: 12 May 2017; Accepted: 15 June 2017; Published: 22 June 2017

\begin{abstract}
The interest in random fiber lasers (RFLs), first demonstrated one decade ago, is still growing and their basic characteristics have been studied by several authors. RFLs are open systems that present instabilities in the intensity fluctuations due to the energy exchange among their non-orthogonal quasi-modes. In this work, we present a review of the recent investigations on the output characteristics of a continuous-wave erbium-doped RFL, with an emphasis on the statistical behavior of the emitted intensity fluctuations. A progression from the Gaussian to Lévy and back to the Gaussian statistical regime was observed by increasing the excitation laser power from below to above the RFL threshold. By analyzing the RFL output intensity fluctuations, the probability density function of emission intensities was determined, and its correspondence with the experimental results was identified, enabling a clear demonstration of the analogy between the RFL phenomenon and the spin-glass phase transition in disordered magnetic systems. A replica-symmetry-breaking phase above the RFL threshold was characterized and the glassy behavior of the emitted light was established. We also discuss perspectives for future investigations on RFL systems.
\end{abstract}

Keywords: random fiber laser; Lévy statistics; photonic spin-glass behavior

\section{Introduction}

Proposals for the operation of random lasers (RLs) were made five decades ago by Ambartsumyan and co-workers [1,2], who visualized the possibility of a new kind of laser that does not require the use of optical cavities. Initially, they reported on the operation of a laser in which one of the cavity mirrors was replaced by a piece of paper that scattered the light in such way that a fraction of the backscattered light was enough to provide feedback for the laser operation. Following the original work, the same group published a series of papers studying the line-narrowing [3], frequency stability [4], and the statistical emission properties [5] of lasers with the so-called nonresonant feedback.

Apparently, the initial motivation for these studies was the observation of laser emission from interstellar media [6], and the interest of the group on this subject continued in the subsequent years [7-9].

For about fifteen years, the majority of research on this theme was pursued by groups that concentrated their effort on the operation of RLs based on microcrystals doped by rare-earth ions [10]. However, the first efficient RL system built in a laboratory environment was reported in 1994 by Lawandy and co-workers [11], who demonstrated the operation of an RL based on dye molecules dissolved in alcohol with suspended titanium dioxide particles. That report was followed by a great number of papers from different authors that investigated other physical systems for efficient RL operation. A large variety of materials have been tested in the past years, and recent publications 
on RLs describe, for example, experiments with dyes dissolved in transparent liquids, gels or liquid crystals with suspended micro or nanoparticles as light scatterers [12-17], powders of semiconductor quantum dots [18,19], dielectric nanocrystals doped with rare-earth ions [20,21], polymers and organic membranes doped by luminescent molecules [22-27], semiconductor and metallic nanowires structures [28-31], and even atomic vapors that present interesting analogies with astrophysical lasers [32].

There is a large literature on RLs motivated by the interest in a deeper understanding of the fundamental properties of RLs [33-36], as well as reports on their possible application in sensing [37], optofluidics [38,39], and imaging [40], among other fields [41]. Moreover, although from a fundamental point of view there are many reports focusing on the basic characteristics of RLs and their operation, the analogies between RLs and other complex systems have only recently been investigated by experiments. For example, in the work by Ghofraniha and co-authors [42], the analogy between RLs and the spin-glass phenomenon typical of highly-disordered magnetic systems was demonstrated for the first time.

In the present work, we review the recent advances on the characteristics of random fiber lasers, which have large potential for applications in various areas, as mentioned below. The article is organized as follows. In Section 2, we describe the Materials and Methods used. In Section 3, the experiments with erbium-doped fibers to characterize the RL behavior and the analysis of the intensity fluctuations are presented, along with the theoretical framework to understand the system behavior. Finally, in Section 4, a summary of the article contents and a discussion on perspectives for future work are presented.

\section{Materials and Methods}

\subsection{Random Fiber Lasers}

Random fiber lasers (RFLs) are akin to RLs, being the one-dimensional (1D) or quasi-1D version of the 2D or 3D RLs. They bear the same nonconventional remarkable characteristic: the optical feedback is provided by a scattering medium, rather than by fixed mirrors or fiber Bragg gratings (FBGs), as in conventional fiber lasers. Similarly to conventional fiber lasers, a gain medium is excited by an appropriate optical pump source.

The first demonstrated RFL, by de Mattos and co-workers in 2007 [43], can be seen as a quasi-1D extension of the colloidal-based RL reported by Lawandy and co-workers in 1994 [11]. In [43], the hollow core of a photonic crystal fiber was filled with a colloid al consisting of Rhodamine $6 \mathrm{G}$ and $250 \mathrm{~nm}$ rutile $\left(\mathrm{TiO}_{2}\right)$ particles suspended in ethylene glicol. By transversely pumping with nanosecond pulses from the second harmonic of a Nd:YAG laser, directional emission was generated axially, and the feedback was due to the $\mathrm{TiO}_{2}$ scatterers. Shortly after the report of ref. [43], Lizárraga and co-authors [44] and Gagné and Kashyap [45] demonstrated the operation of a continuous-wave (CW) pumped erbium-based RFL (Er-RFL), with random FBGs providing the scattering mechanism. We anticipate that this special type of RFL will be exploited as the photonic platform for all of the work described here, and will be detailed later.

A new breakthrough in the research of RFL systems occurred in 2010, when Turitsyn and co-workers [46] first reported on the operation of an RFL system which exploits Rayleigh scattering as the optical feedback mechanism in rather long $(\sim 83 \mathrm{~km})$ conventional single-mode optical fibers. In this pioneer work, the gain mechanism was the stimulated Raman scattering excited in the fiber.

We observe that following this work, the interest in RFL systems and applications has fantastically grown, as reviewed in refs. [47,48]. Indeed, by further exploiting the Rayleigh scattering due to refractive index fluctuations as the mechanism for the multiple light scattering, a myriad of novel types of RFL systems have been demonstrated using a stimulated Raman or Brillouin scattering process.

As most of the works between 2007 and 2014 have been reviewed in refs. [47,48], including polymer-based optical fibers or plasmonically-enhanced RFLs, we highlight here the diversity of 
works reported over the years 2015 and 2016 (see [49-66] and references therein). As examples, we mention that a Q-switched operation has been reported using Brillouin scattering [56], with pulses as short as $42 \mathrm{~ns}$ at $100 \mathrm{kHz}$ being demonstrated. Regarding the fiber length, since the first observation of RFL made using conventional fibers with $83 \mathrm{~km} \mathrm{[46],} \mathrm{RFLs} \mathrm{with} \mathrm{fiber} \mathrm{lengths} \mathrm{as} \mathrm{short}$ as $120 \mathrm{~m}$ have been reported [58], also providing $200 \mathrm{~W}$ of output power. Other recent features of RFLs include tunability using graphene-based devices [56] or high-order Raman scattering [57], second harmonic generation [59], polarized emission from disordered polymer optical fibers [60], and photonic turbulence [67].

\subsection{Fiber Bragg Grating-Based Random Fiber Lasers}

As mentioned above, FBG-based RFLs were first introduced in 2009 [44,45]. Currently, the FBG fabrication methods, characterization, and management constitute a well-developed field, and further information on this subject is deferred to ref. [68].

Typically, a writing setup based, for instance, on CW UV radiation or femtosecond sources at $800 \mathrm{~nm}$, is employed to inscribe an FBG into a conventional or core-doped optical fiber. The case of interest here exploits an active core single-mode fiber, using trivalent erbium ions, $\mathrm{Er}^{3+}$, which can be excited at $980 \mathrm{~nm}$ or $1480 \mathrm{~nm}$, and emits in the 1540-1560 nm spectral region. Instead of inscribing the FBGs in an evenly spaced way, thus leading to conventional resonators, the erbium-based FBGs are randomly spaced and play the role of random scatterers, leading to RFL emission. Generally, the fiber is placed on a movable stage which is randomly displaced, thus providing the randomness in the FBG writing process. Several tens to hundreds of gratings can be inscribed along several tens of $\mathrm{cm}$ of fiber length.

In the next section, we will describe the fabrication and characterization of the Er-RFL system with a specially-designed FBG [45]. We comment that this system has been lately used as an experimental platform to study complex photonic phenomena, such as the observation of unconventional Lévy-like statistics of output intensity values and the demonstration of the nontrivial replica-symmetry-breaking regime, which marks the signature of the phase transition from a photonic paramagnetic to a photonic spin-glass phase.

\section{Results}

\subsection{Characterization of the FBG-Based Er-RFL Explored as a Statistical-Physics Experimental Platform}

As reported by Gagné and Kashyap [45], a unique FBG was produced by writing an exceptionally high number of gratings ( $>1000$ ) over a $30 \mathrm{~cm}$ length fiber. A polarization-maintaining erbium-doped fiber from CorActive (peak absorption $28 \mathrm{~dB} / \mathrm{m}$ at $1530 \mathrm{~nm}$, NA 0.25 , mode field diameter $5.7 \mu \mathrm{m}$ ) was employed, in which the randomly distributed phase errors grating was written, instead of a random array of gratings as in [44]. It was realized in [45] that during the movement of the translation stage, the friction between the fiber and the mount introduced irregularities in the grating spectrum that could be controlled by managing the air flow from the vacuum. Such irregularities were perceived as small phase errors, randomly but continuously distributed along the grating profile being inscribed. Thanks to this procedure, a high number of modes were observed, which is very important for several applications, as we will see later.

The Er-RFL described in [45] had a very low threshold of $3 \mathrm{~mW}$, with a throughput efficiency of $\sim 4.5 \%$ for $100 \mathrm{~mW}$ pump power. The number of emitted modes was dependent on the pump power and fiber length ( 20 or $30 \mathrm{~cm}$ fiber lengths were characterized in [45]), and a single or few modes were observed, limited by the system measurement resolution [69].

For the experiments described here, the $30 \mathrm{~cm}$ long fiber was employed with the experimental setup shown in Figure 1, reproduced from ref. [68]. The pump source was a semiconductor laser operating in the CW regime at $1480 \mathrm{~nm}$, delivering $150 \mathrm{~mW}$ output power at the fiber pigtail. The Er-RFL output was split, through a $1480 \mathrm{~nm} / 1550 \mathrm{~nm}$ wavelength-division multiplexer (WDM), 
with a split ratio of 10/90 for $1480 \mathrm{~nm}$ and $1550 \mathrm{~nm}$, respectively, to a power meter and a spectrometer. The employed fiber splices were lossy, if compared to the original work [45], leading to a higher threshold. The spectrometer (SpectraPro 300i, Acton Research, Acton, MA, USA), coupled to a liquid- $\mathrm{N}_{2}$ cooled InGaAs CCD camera, had a nominal resolution of $0.1 \mathrm{~nm}$.

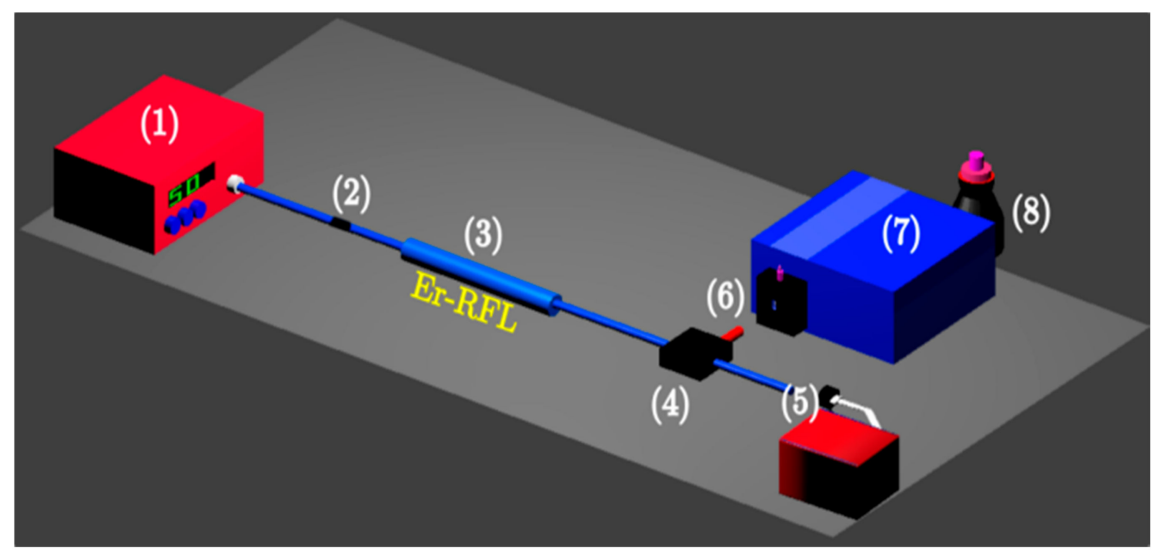

Figure 1. Experimental setup for the erbium-based random fiber laser (Er-RFL) system. (1) Fiber pigtailed semiconductor laser operating in the continuous-wave $(\mathrm{CW})$ regime. (2) Fiber connector. (3) Er-doped RFL. (4) Wavelength-division multiplexer (WDM) 1480-1550. (5) Power meter to measure the output power $P_{\text {out }}$ at $1480 \mathrm{~nm}$. (6) RFL emission out to the spectrometer. (7) Spectrometer. (8) Liquid- $\mathrm{N}_{2}$ cooled InGaAs CCD camera. (Reproduced with permission from ref. [68]).

Figure 2a shows the Er-RFL output spectrum for intensities below and above the RFL threshold, while Figure $2 b$ shows the linewidth narrowing (left y-axis) and emitted Er-RFL intensity (right y-axis) as a function of the pump power $P$ normalized to the threshold power $P_{t h}$. The threshold power was $P_{t h}=16.30 \pm 0.05 \mathrm{~mW}$ [68], which is higher than in the original work of ref. [45] due to the lossy components employed. However, this fact did not affect the experimental studies, and the output was typically around $1-2 \mathrm{~mW}$.
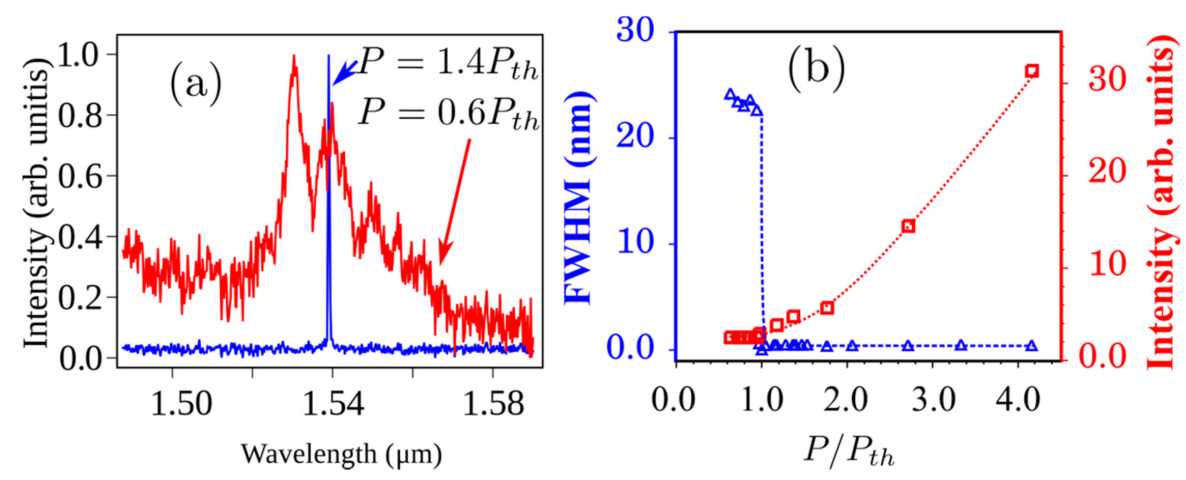

Figure 2. (a) Emitted spectrum of Er-RFL before (red) and after (blue) the laser threshold; (b) Emitted intensity (squares) and FWHM (triangles) of the Er-RFL system as a function of the normalized input power. The measured threshold power was $P_{t h}=16.30 \pm 0.05 \mathrm{~mW}$. The dotted lines are a guide to the eyes. (Reproduced with permission from ref. [68]).

Besides the routine characterization illustrated in Figure 2, two other analyzes were performed in order to show that the laser is multimode, i.e., with many longitudinal modes, and that the intensity fluctuations do not depend on the pump laser fluctuations.

First, in order to demonstrate the multimode characteristic of the Er-RFL, we employed the technique of speckle contrast [69], following the work of refs. [40,70,71]. To generate the speckle, 
a scattering medium with dried $\mathrm{TiO}_{2}(250 \mathrm{~nm})$ nanoparticles in water solution on a microscope slide along with a Kohler illumination system was used. Different CCD cameras were employed for the data acquisition, depending upon the source wavelength in the visible or near-infrared. The relation between the speckle contrast $C$ and the number $m$ of longitudinal modes of the source is given by [71] $C=\sigma /\langle I\rangle=1 / \sqrt{m}$, where $\sigma$ and $\langle I\rangle$ are the standard deviation and the average intensity determined from the speckle, respectively. Figure 3 shows the obtained results for different light sources.

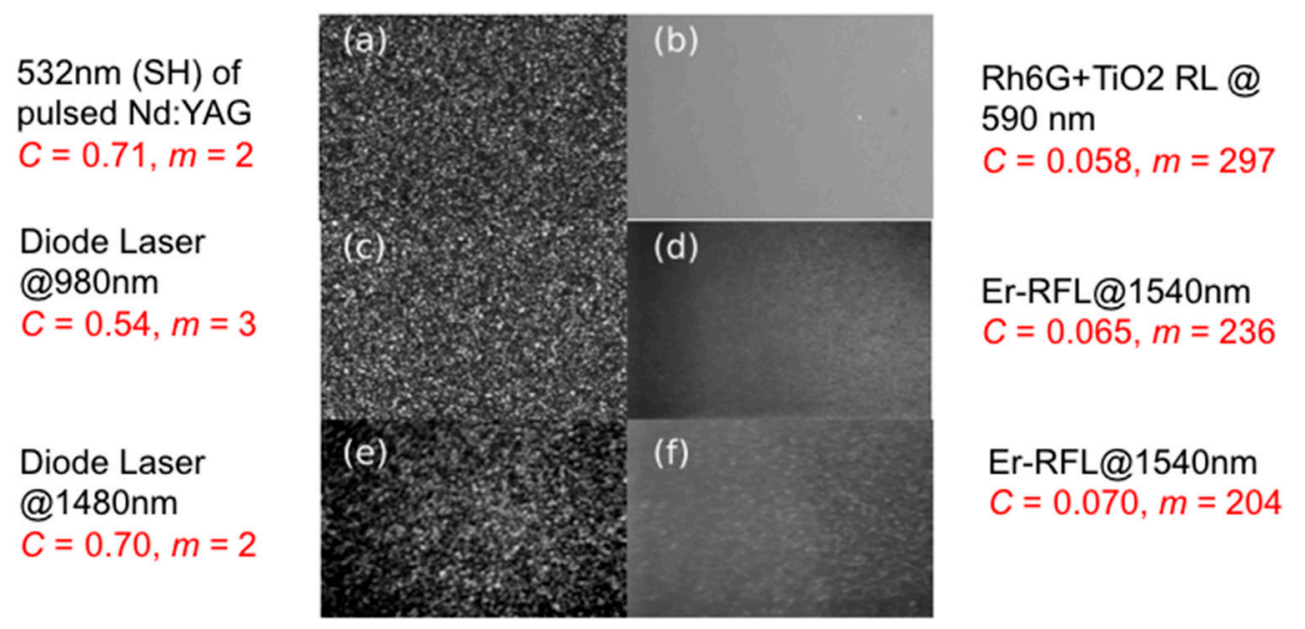

Figure 3. (a,c,e) display the measured speckle of the second harmonic of a pulsed Nd:YAG laser (a); a CW diode laser operating at $980 \mathrm{~nm} \mathrm{(c);} \mathrm{and} \mathrm{a} \mathrm{similar} \mathrm{diode} \mathrm{laser,} \mathrm{but} \mathrm{operating} \mathrm{at} 1480 \mathrm{~nm}(\mathbf{e})$; $(\mathbf{b}, \mathbf{d}, \mathbf{f})$ show a colloid random laser (RL) (b); the Er-RFL at $1540 \mathrm{~nm}$ pumped at $980 \mathrm{~nm}(\mathbf{d})$; and the same Er-RFL pumped at $1480 \mathrm{~nm}$ (f). The values of the speckle contrast $C$ and number of longitudinal modes $m$ for each optical source are indicated at the side. (Adapted with permission from ref. [69]).

To confirm the experimental results for the Er-RFL system, we initially characterized a well-known colloidal-based RL consisting of Rhodamine $6 \mathrm{G}$ and $\mathrm{TiO}_{2}$ nanoparticles. The pump source was the second-harmonic of a pulsed Nd:YAG laser (Ultra, BigSky Laser, Paris, France), operating at $5 \mathrm{~Hz}$ and delivering pulses of $\sim 7 \mathrm{~ns}$. Figure $3 \mathrm{a}$ shows the speckle image from the pump source, which is a highly coherent source (basically a single mode), and Figure $3 \mathrm{~b}$ shows the equivalent image from the colloidal RL. Just as in the work of refs. [40,70], the speckle-free RL emission is corroborated, and the calculated values of the parameters $C$ and $m$ for the RL are 0.058 and 297, respectively, which are very much distinct from the respective values obtained for the pump laser. Additionally, the speckle images from Figure $3 \mathrm{a}, \mathrm{b}$ are strikingly different, as already reported [40].

The same behavior is reproduced for the Er-RFL system, in which the pump laser at $1480 \mathrm{~nm}$ presents $m=2$ longitudinal modes (Figure 3e), whereas the Er-RFL displays the presence of $m=204$ longitudinal modes (Figure 3f). For completeness, the pump semiconductor laser at $980 \mathrm{~nm}$ was also employed, giving similar results, i.e., a $980 \mathrm{~nm}$ pump laser was a quasi-single mode ( $m=3$, Figure 3c), whereas the Er-RFL system showed $m=236$ longitudinal modes, as illustrated in Figure 3d. Actually, being a multimode system is a fundamental requirement for the observation of the spin-glass type of behavior in RLs, as discussed below.

On the other hand, we also remark that the fluctuations of the pump source (less than $5 \%$ ) were not correlated with the RFL fluctuations, as similarly demonstrated in [42,72]. This important point is corroborated by the results displayed in Figure $4 \mathrm{a}, \mathrm{b}$, showing, respectively, the spectral variance of the Er-RFL system and the normalized standard deviation of both the pump laser and Er-RFL. It is thus clear from Figure $4 \mathrm{~b}$ that the pump laser fluctuations do not affect the Er-RFL fluctuations, particularly because the pump laser was kept working all the time well above the threshold, so that the kind of new physics observed around the threshold in the Er-RFL would not be detected, even if present. 

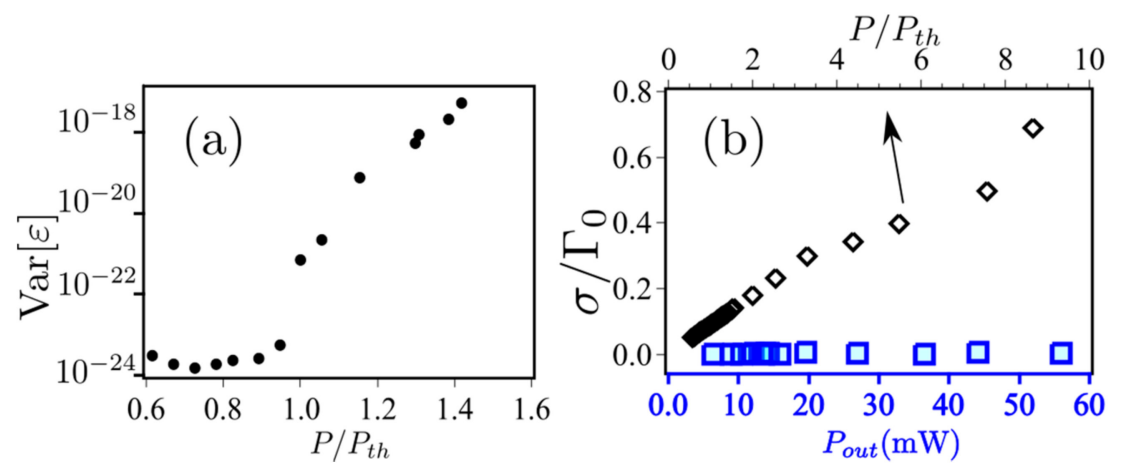

Figure 4. (a) Variance of the emitted intensity as a function of the normalized input power $P / P_{t h}$; (b) Standard deviation of the maximum intensity (normalized by its average value) of the $\mathrm{CW}$ semiconductor pump laser (as function of the output power $P_{\text {out }}$; squares) and the Er-RFL system (as function of the normalized input power $P / P_{\text {th }}$; diamonds). (Reproduced with permission from ref. [68]).

\subsection{Theoretical Framework}

A great advance in the theoretical understanding of the combined effect of amplification, nonlinearity, and disorder in RL systems was put forward in a series of articles [73-82] published within the last decade. In this subsection, we review the physical mechanisms and some analytical developments underlying the richness of photonic behaviors displayed in the phase diagram of RL systems, as a function of the input excitation power and disorder strength. In fact, in refs. [73-80], a variety of interesting photonic phases emerge, which keep close analogies with some characteristic behaviors of magnetic systems, such as spin glass, paramagnetism, and ferromagnetism.

Moreover, such richness is also present in the diversity of statistical regimes observed in the output intensity emitted by RL systems. Interestingly, as we shall see below, the same theoretical starting point that gives rise to the variety of photonic behaviors also explains the shifts in the statistical properties of the distribution of intensity values. In fact, as discussed in the following, the increasing of the excitation power promotes a sequence of changes in the distribution of intensity values, from a Gaussian regime below the RL threshold to a Lévy-type behavior around the RL transition, and back to a second Gaussian regime well above the threshold. The theoretical basis $[81,82]$ for these statistical aspects of RL emission is also reviewed below.

We start by reviewing the theoretical background [73-80] underlying the diverse photonic behaviors displayed by RLs. In a photonic system with intrinsic disorder, arising either from the inherent optical random noise or from the presence of randomly-located light scatterers (e.g., micro or nanoparticles [12-17], or dielectric nanocrystals doped with rare-earth ions [20,21]), the amplitudes $a_{k}$ of the electromagnetic modes generally present stochastic dynamics. The Langevin approach is thus a suitable theoretical framework to describe such dynamics through the following set of equations,

$$
\frac{d a_{k}}{d t}=-\frac{\partial H}{\partial a_{k}^{*}}+F_{k}
$$

where $F_{k}$ represents a Gaussian (white) uncorrelated optical noise term, and in the slow-amplitude regime of the modes, the general complex-valued functional $H$ is given by [73-80] (closely following the notation of [78]):

$$
H=\sum_{\left\{k_{1} k_{2}\right\}^{\prime}} g_{k_{1} k_{2}}^{(2)} a_{k_{1}} a_{k_{2}}^{*}+\frac{1}{2} \sum_{\left\{k_{1} k_{2} k_{3} k_{4}\right\}^{\prime}} g_{k_{1} k_{2} k_{3} k_{4}}^{(4)} a_{k_{1}} a_{k_{2}}^{*} a_{k_{3}} a_{k_{4}}^{*}
$$

The symbol $\{\ldots\}^{\prime}$ implies the frequency-matching conditions $\left|\omega_{k_{1}}-\omega_{k_{2}}\right|<\gamma$ and $\left|\omega_{k_{1}}-\omega_{k_{2}}+\omega_{k_{3}}-\omega_{k_{4}}\right|<\gamma$ in the quadratic and quartic terms, respectively, with $\gamma$ denoting the 
finite linewidth of the modes. The disordered nature of the scattering medium directly affects both the quadratic and quartic interactions among the spatially-overlapping modes in Equation (2). Indeed, the physical origin of the quadratic coupling $g_{k_{1} k_{2}}^{(2)}$ lies in the spatially inhomogeneous refractive index, as well as in the nonuniform distribution of the gain and effective damping contribution due to the cavity leakage. In systems with null or weak leakage, in which the off-diagonal contribution in $g_{k_{1} k_{2}}^{(2)}$ is negligible, the coefficient rates of amplification $\left(\gamma_{k}\right)$ and radiation loss $\left(\alpha_{k}\right)$ are related to the real part of the diagonal coupling through $g_{k k}^{(2) R}=\operatorname{Re}\left\{g_{k k}^{(2)}\right\}=\alpha_{k}-\gamma_{k}$. On the other hand, the quartic coupling $g_{k_{1} k_{2} k_{3} k_{4}}^{(4)}$ is associated with the modulation of the nonlinear $\chi^{(3)}$-susceptibility with a random spatial profile [73-80].

The spatial disorder in the scattering medium generally makes the explicit calculation of the quadratic and quartic couplings in Equation (2) rather difficult. Consequently, in [73-80], these couplings were considered as quenched Gaussian variables, with probability distributions independent of the mode combinations $\left\{k_{1} k_{2}\right\}^{\prime}$ and $\left\{k_{1} k_{2} k_{3} k_{4}\right\}^{\prime}$, respectively. In addition, in the mean-field approach of refs. [73-80], the above frequency-matching constraints were relaxed, implying that all modes interact unrestrictedly. In the case in which the total optical intensity, $I=\sum_{k} c_{k}\left|a_{k}\right|^{2}$, is a constant, with time-independent prefactors $c_{k}$, the real part $H^{R}$ of the Functional $(2)$ is shown in $[78,80]$ to become analogous to the Hamiltonian of the magnetic $p$-spin model with a spherical constraint [83]. Indeed, the $p$-spin Hamiltonian also presents a sum of quadratic $(p=2)$ and quartic $(p=4)$ interaction terms, just as in Equation (2), with the couplings drawn from Gaussian distributions [83]. Moreover, in the $p$-spin model, the sum of the squared spin variables is a constant (spherical constraint), as also happens to the total optical intensity $I$ in the photonic system. This photonic-to-magnetic analogy is indeed relevant, since it allows identifying the amplitudes of the modes with the spin variables, and the excitation (pump) energy in the photonic system with the inverse temperature in the magnetic one. In this sense, we comment below that the typical magnetic phases exhibited by the disordered $p$-spin model can also find an analogous counterpart in the phase diagram of an RL system.

Once the disordered Hamiltonian $H^{R}$, given by the real part of Equation (2), has been built in terms of mode amplitudes that are spin analogues, a repertoire of statistical-physics-based analytical techniques to treat disordered magnetic systems becomes immediately available to the photonic system. In particular, the so-called replica trick [84] can be readily applied to $H^{R}$. This approach essentially consists of considering identical copies (i.e., replicas) of the system in order to compute the powers $Z^{n}$ of the partition function, while calculating the free energy from the limit expression $\ln Z=\lim _{n \rightarrow 0}\left(Z^{n}-1\right) / n$. At the end, the phase diagram of the RL system is obtained as a function of the input pumping rate and disorder strength [77-79]. Remarkably, the physical equivalence (or symmetry) among these replicas can be broken in some circumstances, as discussed below.

Following this photonic-to-magnetic analogy, the photonic phases identified in the RL system maintain some resemblance to the magnetic behaviors of the $p$-spin model. Indeed, we next describe the main properties of the four photonic regimes obtained in the phase diagram of refs. [77-79], namely: incoherent wave, mode-locking laser, phase-locking wave, and spin-glass RL behavior.

In the incoherent-wave regime, which occurs for low input powers and any disorder strength, the modes oscillate incoherently in an uncorrelated way. In this case, the system operates in a regime with amplified spontaneous emission. According to the analogy above, this phase is similar to the paramagnetic behavior in spin systems at high temperatures and for any degree of disorder, in which the uncorrelated spin directions are random and present fast dynamics. Moreover, just as in the paramagnetic phase, the incoherent-wave solution preserves the symmetry among the photonic replicas (see also below).

The mode-locking laser behavior presents modes oscillating coherently with the same phase, at high input powers, without disorder or for low degrees of disorder. It corresponds to the ferromagnetic phase in spin systems at low temperatures, either without disorder, in which all spins align parallel, or in the presence of low disorder, as in the case of the random bond ferromagnet, with a few clusters of disordered spins in a predominantly ferromagnetic background. 
Remarkably, the regime described as the phase-locking wave has no perfect analogous counterpart in disordered spin modes. In the pumping rate versus disorder strength diagram, it occupies an intermediate region between the incoherent-wave and spin-glass RL phases. In this regime, the mode phases are only partially locked.

Finally, in the RL regime, obtained for input powers above the threshold and in the presence of strong disorder, the synchronous oscillation of the modes is frustrated (in contrast with the mode-locking laser behavior), and they acquire phase coherence and nontrivial correlations (differently from the incoherent-wave regime). In this case, the analogue magnetic phase is the spin-glass regime observed in highly disordered magnetic systems at low temperatures, in which the spins point at random directions, while being strongly correlated in time and with rather slow (frozen) dynamics. In the photonic, as well as in the magnetic spin-glass phase, the replicas undergo a nontrivial breaking of symmetry, which is explained as follows.

The concept of replica symmetry breaking (RSB) was introduced by G. Parisi in 1979 in the context of the theory of disordered magnetic systems [84]. In this framework, for sufficiently low temperatures and strong disorder (e.g., in the spin couplings or locations), the free energy landscape breaks into a large number of local minima in the configuration space. Due to the frustrated magnetic interactions in the disordered Hamiltonian, the spins fail to align in a spatially regular configuration, as in the ferromagnetic state. Instead, spins "freeze" along random directions, with rather slow dynamics, in a spin-glass state. As a given spin configuration can be trapped for a long time in a local free energy minimum, metastability and irreversibility effects arise in the spin-glass phase, e.g., magnetic hysteresis. Consequently, identical systems, with the same distribution of spin interactions and prepared under identical conditions (i.e., replicas of the spin system), can reach rather distinct states that lead to different measures of observable quantities and nontrivial correlation patterns. In this case, the system replicas are no longer physically equivalent (or symmetric), and an RSB scenario emerges. Later on, the scope of the concept of RSB was much extended to reach other complex systems [84], including neural networks and structural glasses.

In order to identify a regime with RSB, it becomes necessary to calculate a correlation function that gives a measure of the overlap between two given replicas [84]. In the case of magnetic systems, the replica overlap parameter is a spin-spin correlation function defined by the product of a certain spin occupying the same position in two distinct replicas. In the sequence, the sum of all spins is performed. By considering each pair of replicas, a distribution $\mathrm{P}(q)$ of values of such an overlap parameter $q$ is thus obtained. If this distribution is centered around zero, the replicas are considered symmetric, a scenario that is observed in the paramagnetic phase in spin systems. However, if the distribution peaks at non-zero values of the replica overlap parameter, then the symmetry of replicas is broken, and an RSB spin-glass phase can emerge. Therefore, in this sense, a parameter $q_{\max }$ can be defined to indicate the locus of the maximum of the distribution $\mathrm{P}(q)$, which is considered as the Parisi order parameter [84]. The value of $q_{\max }$ thus signalizes a replica-symmetric paramagnetic or an RSB spin-glass phase, respectively, if the maximum of $\mathrm{P}(q)$ occurs exclusively at $q_{\max }=0$ (no RSB) or also at values $\left|q_{\max }\right| \neq 0$ (RSB).

In the photonic context, an analogue correlation function between modes can also be suitably defined [42,79] (see details below). Its distribution of values determines, in a similar way, the presence or absence of the photonic RSB spin-glass phase.

On the experimental side, the very first evidence of photonic RSB glassy behavior in an RL system arose in the 2D functionalized $\mathrm{T}_{5} \mathrm{OC}_{\mathrm{x}}$ oligomer amorphous solid-state material [42]. Subsequent demonstrations appeared in 3D functionalized $\mathrm{TiO}_{2}$ particle-based dye-colloidal [85] and neodymium-doped $\mathrm{YBO}_{3}$ solid-state [82] RLs. Here, we highlight that the RSB spin-glass phase has also been characterized in the above-mentioned Er-RFL system [68,69]. The emergence of such behavior in Er-RFL is actually justified since this system also presents the disorder and nonlinear ingredients necessary to induce the RSB glassy RL phase, as discussed above in the context of the effective photonic Hamiltonian (2). 
We now turn to the discussion on the statistical regimes of output intensities emitted by RL systems.

We first review some analytical developments regarding the distribution of intensity values. Noteworthy, the set of Langevin equations, given by Equation (1), also provides the underlying theoretical basis for such analysis. Indeed, by writing $I_{k}=c_{k}\left|a_{k}\right|^{2}$, a manipulation of Equation (1) yields [82]:

$$
\frac{1}{c_{k_{2}}} \frac{d I_{k_{2}}}{d t}=-2 \operatorname{Re}\left\{\sum_{\left\{k_{1}\right\}^{\prime}} g_{k_{1} k_{2}}^{(2)} a_{k_{1}} a_{k_{2}}^{*}+\frac{1}{2} \sum_{\left\{k_{1} k_{3} k_{4}\right\}^{\prime}}\left[g_{k_{1} k_{2} k_{3} k_{4}}^{(4)}+g_{k_{1} k_{4} k_{3} k_{2}}^{(4)}\right] a_{k_{1}} a_{k_{2}}^{*} a_{k_{3}} a_{k_{4}}^{*}+a_{k_{2}}^{*} F_{k_{2}}\right\}
$$

The restricted sum in the quartic coupling generally involves three classes of mode: combinations [73,86]: $\omega_{k_{1}}=\omega_{k_{2}}$ and $\omega_{k_{3}}=\omega_{k_{4}}, \omega_{k_{1}}=\omega_{k_{4}}$ and $\omega_{k_{2}}=\omega_{k_{3}}$, and the remaining possibilities satisfying the frequency-matching conditions, which have been usually disregarded $[73,86]$. We consider the diagonal contribution in the quadratic coupling to dominate over the off-diagonal part. By expressing the optical white noise as the sum of additive and multiplicative statistically independent stochastic processes [87], so that $F_{k}(t)=F_{k}^{(0)}(t)+a_{k}(t) F_{k}^{(1)}(t)$, and considering slow-amplitude modes $a_{k}(t)$ (if compared to the rapidly evolving phase dynamics), we obtain the Fokker-Planck equation $[81,82,87]$ for the probability density function (PDF) of the output intensity,

$$
\frac{\partial P}{\partial t}=-\frac{\partial}{\partial I_{k}}\left[\left(-d_{k} I_{k}-b_{k} I_{k}^{2}+2 Q I_{k}\right) P\right]+2 Q \frac{\partial^{2}}{\partial I_{k}^{2}}\left(I_{k}^{2} P\right)
$$

where the parameter $Q$ controls the magnitude of the multiplicative fluctuations through: $\left[F_{k}^{(1) R}(t) F_{m}^{(1) R}\left(t^{\prime}\right)\right]=2 Q \delta_{k, m} \delta\left(t-t^{\prime}\right), b_{k}=g_{k k k k}^{(4) R} / c_{k}$, and

$$
d_{k}=\sum_{n \neq k}\left[g_{k k n n}^{(4) R}+g_{k n n k}^{(4) R}+g_{n k k n}^{(4) R}+g_{n n k k}^{(4) R}\right] I_{n} / c_{n}-2\left(\gamma_{k}-\alpha_{k}\right)
$$

The steady-state solution of Equation (4) is [81,82,87]:

$$
P\left(I_{k}\right)=A_{k} I_{k}^{-\mu_{k}} \exp \left(-b_{k} I_{k} / 2 Q\right)
$$

with $I_{k}>0, A_{k}$ as the normalization constant, and $\mu_{k}=1+d_{k} / 2 Q$. This PDF presents a power-law decay combined with exponential attenuation. Its second moment can be very large, though still finite, depending on the value of $\mu_{k}$, mainly if $b_{k} / 2 Q \ll 1$. Indeed, the experimental results obtained for the Er-RFL (see below) indicate [68] that the distribution of output intensities displays much larger variance and much stronger fluctuations close to the threshold, if compared with those below and above the threshold. In this sense, as argued below, the PDF of intensities is most properly described by the Lévy $\alpha$-stable distribution [88] for long time measurements performed in CW pumped RLs, such as the Er-RFL system [68], or during an extensive number of shots in the case of pulsed RLs [89].

As we now turn the focus to the physical discussion on the PDF of intensity values, Equation (6), we initially observe that the analysis is strictly connected with the central limit theorem (CLT) and generalized CLT of statistics. These theorems determine the attraction of the PDF of the sum of a large number of random variables to one of the possible asymptotic stable distributions, namely the Gaussian or the Lévy $\alpha$-stable family [88]. In the present photonic context, if the stochastic values assumed by the intensity $I$ are identically distributed and uncorrelated over the long sequence of output spectra (or even if they present finite-time correlations), and if the second moment of the PDF $P(I)$ is finite, then the CLT assures [88] that the intensity fluctuations are driven by the Brownian (Gaussian, normal) dynamics. On the other hand, if the second moment of $P(I)$ diverges, the generalized CLT states [88] that the fluctuations are asymptotically governed by the Lévy statistics. The continuous 
family of Lévy $\alpha$-stable distribution is described [88] by the Fourier transform of the characteristic function defined in $k$-space,

$$
\bar{P}(k)=\exp \left\{-|c k|^{\alpha}[1-i \beta \operatorname{sgn}(k) \Phi]+i k v\right\} .
$$

The Lévy index $\alpha \in(0,2]$ is the most important parameter, since it drives the magnitude of the intensity fluctuations. Indeed, whereas strong fluctuations with relevant deviations from the Gaussian behavior are associated with values in the range $0<\alpha<2$, the Gaussian statistics with relatively weak fluctuations and the result of the CLT are recovered for the boundary value $\alpha=2$. Therefore, Equation (7) can suitably describe both Gaussian and Lévy statistical regimes, depending only on the value of the single parameter $\alpha$. In other words, the parameter $\alpha$, which can be experimentally determined from the direct analysis of the PDF $P(I)$, effectively works as an indicator of the statistical regime (Gaussian or Lévy) of intensity fluctuations. The other independent parameters describe the asymmetry or skewness of the distribution $(\beta \in[-1,1])$, location $(v \in(-\infty, \infty))$, and scale $(c \in(0$, $\infty)$ ), along with the function $\Phi(k)=-(2 / k) \ln |k|$ if $\alpha=1$, whereas $\Phi=\tan (\pi \alpha / 2)$ if $\alpha \neq 1$.

Though the Lévy PDF, given by the Fourier transform of Equation (7), displays closed analytical form only for a few values of $\alpha$ (e.g., the Cauchy distribution arises for $\alpha=1$ and $\beta=0$ ), its large- $I$ asymptotic behavior is power-law tailed, $P(I) \sim I^{-\mu}$, with exponent $\mu=1+\alpha$. Conversely, it is also true that random variables with power-law distribution are governed by the Lévy PDF with $\alpha=\mu-1$ if $1<\mu<3$ (diverging second moment), and by the $\alpha=2$ Gaussian statistics if $\mu \geq 3$ (finite second moment) [88]. Therefore, if the PDF of intensities presents asymptotic power-law behavior, then the power-law exponent $\mu$ also indicates the type of statistical regime (Gaussian or Lévy) of the output intensity values.

At this point, some words of caution are necessary in order to properly interpret the actual experimental data of the Er-RFL system under the statistical framework of the CLT and generalized CLT.

We initially remark that in the present case of intensity fluctuations, as well as in any case of realistic stochastic phenomena, a PDF with a diverging second moment actually represents an unphysical possibility. Nevertheless, it has been demonstrated [90] that a truncated power-law PDF, with a large but finite second moment, behaves rather similarly to the Lévy PDF to a considerable extent, defining the so-called Lévy-type (or Lévy-like) statistical behavior. In this case, the crossover to the Gaussian dynamics, predicted by the CLT, is only attained in a very long term [90,91]. In this sense, theoretically justified truncation schemes have been suitably implemented, for example, by restricting the values of the random variable to a finite range [90,91], with $P(I)=0$ for $I>I_{\text {cutoff }}$, or by tempering the power law with an exponential attenuation [81,82,89], $P(I) \sim \exp (-\eta I) / I^{\mu}$, in a form similar to Equation (6). Therefore, the experimental reports of Lévy PDFs of intensities with index $0<\alpha<2$ should be properly interpreted as representative of this extensive Lévy-like statistical regime of intensity measurements.

Lastly, in addition to the description above of the truncated power-law with exponential attenuation, which was based on the Langevin dynamics of the amplitudes of the normal modes, we also comment that a PDF of output intensities emitted by RL systems with power-law form, $P(I) \sim I^{-\mu}$, has been derived in [92]. In contrast with the above developments, in this case, the theoretical approach took into account the statistics of the photon trajectories subjected to multiple scatterings within the sample [92]. The power-law exponent was found to be $\mu=1+\ell_{\mathrm{g}} /\langle l\rangle$, where $\ell_{\mathrm{g}}$ and $\langle l\rangle$ denote, respectively, the gain length of the active medium and the average length of the photon paths.

\subsection{Lévy Statistics and Glassy Behavior in Er-RFL}

In this subsection, we focus on the statistical analysis of the experimental data of the Er-RFL system. 
We start by analyzing the intensity spectra. Figure 5a-c display 5000 spectra for each input power, from which the intensity fluctuations can be appreciated in the regimes below (Figure 5a), around (Figure 5b) and above (Figure 5c) the RL threshold [68]. According to the discussion in Section 3.2, the strong intensity fluctuations observed near the threshold suggest that the PDF of the output intensities can be described by the family of Lévy $\alpha$-stable distributions, including the Lévy statistical regime if $0<\alpha<2$ and the Gaussian limit if $\alpha=2$.

Figure $5 \mathrm{~d}-\mathrm{f}$ portrays the distributions $P(I)$ obtained from the data of Figure $5 \mathrm{a}-\mathrm{c}$, as well as the respective best fits to Equation (7) by applying the quantile-based method $[93,94]$. Best-fit values of the parameters are summarized in Table 1 . The values of $\alpha$ are consistent with the Gaussian profiles $(\alpha=2.0)$ shown in Figure $5 \mathrm{~d}, \mathrm{f}$, respectively, below and above the threshold, and also with the Lévy-like $\operatorname{PDF}(\alpha=1.3)$ around the threshold, observed in Figure 5e. The unit value of $\beta$, indicating the maximum skewness of the distribution, in all cases reflects the asymmetry related to the positiveness of the intensity. Furthermore, the values of the location parameter $v$ in the Gaussian regimes, $v=0.858$ for $P / P_{t h}=0.6$ and $v=0.682$ for $P / P_{t h}=1.8$, agree with the mean values, respectively, observed in Figure $5 \mathrm{~d}$,f. Indeed, the actual Gaussian distributions, which are equivalent to the $\alpha=2$ Lévy PDFs in Figure $5 \mathrm{~d}$,f, present the mean $v$ and standard deviation $\sqrt{2} c$, as theoretically predicted [88]. Interestingly, when comparing the Gaussian regimes below and above the threshold, we notice a considerable broadening of the PDF $P(I)$ at $P / P_{\text {th }}=1.8$, leading to a wider spread of intensities (Figure $5 \mathrm{f}$ ). This result is associated with the larger second moment of the distribution and more intense fluctuations observed above the threshold. Those fluctuations still remain, however, much weaker than the ones measured in the crossover region.
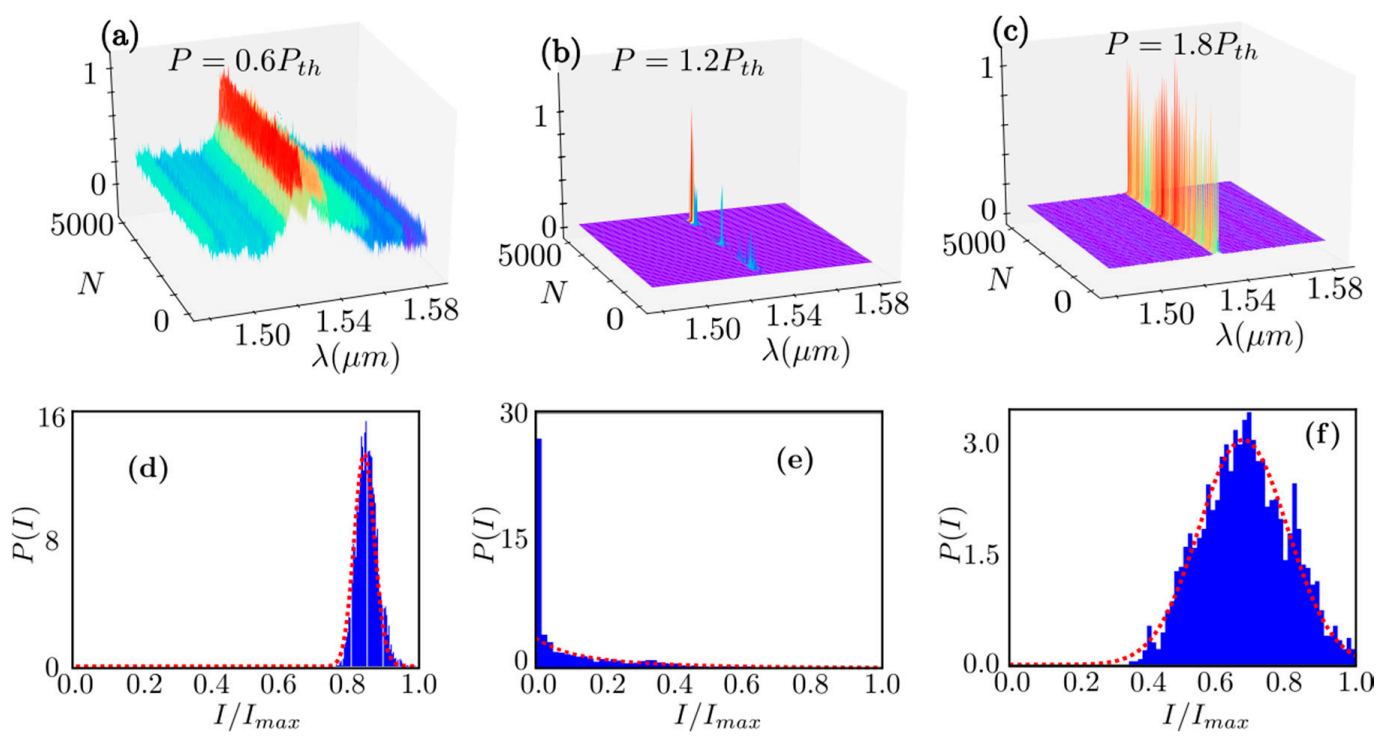

Figure 5. (a-c) 5000 intensity spectra of the Er-RFL system for each input power (a) below; (b) around; and (c) above the RL threshold; (d-f) Probability density functions (PDFs) $P(I)$ of maximum intensities obtained from the data shown in (a-c). The best fits using Equation (7) are depicted in dotted lines and portrait Gaussian profiles (d) below and (f) above the threshold $(\alpha=2.0)$; and (e) a Lévy distribution $(\alpha=1.3)$ around the threshold. (Reproduced with permission from ref. [68]).

Table 1. Summary of best fit parameters to Equation (7) for the intensity distributions of Figure 5d-f [68].

\begin{tabular}{ccccc}
\hline Input Excitation Power & $\boldsymbol{\alpha}$ & $\boldsymbol{\beta}$ & $\boldsymbol{c}$ & $\boldsymbol{v}$ \\
\hline$P / P_{\text {th }}=0.6$ & 1.9 & 1.0 & 0.021 & 0.858 \\
$P / P_{\text {th }}=1.2$ & 1.3 & 1.0 & 0.061 & -0.193 \\
$P / P_{\text {th }}=1.8$ & 2.0 & 1.0 & 0.091 & 0.682 \\
\hline
\end{tabular}


The main result confirming the Lévy behavior of the output intensities of the Er-RFL system is shown in Figure 6. With a basis on ref. [68], we first notice that the variation of the Lévy index $\alpha$ as a function of the normalized input power clearly points to the presence of three distinct statistical regimes. Indeed, as $P / P_{\text {th }}$ increases, the statistics of the output intensities progressively shift from the prelasing Gaussian $(\alpha=2)$ to the Lévy $(0<\alpha<2)$ behavior around the threshold, and to the subsequent Gaussian $(\alpha=2)$ regime deep in the RL phase. Noticeably, this sequence also resembles the statistical behavior of the output intensity of 3D bulk RLs $[82,89,93,95,96]$. We also notice that, as pointed in ref. [93], the second Gaussian regime is rather distinct from the first one. Indeed, in the second Gaussian phase above the RL threshold, the system is in the regime with self-averaging of the gain [93]. In contrast, in the first Gaussian phase, it is still in the prelasing regime. Moreover, we also comment that the observed independence on the spatial dimensionality of the Lévy character of intensity fluctuations finds support in the theoretical analysis based on Langevin equations, previously discussed in Section 3.2, which is considered to hold, irrespective of the spatial dimension [69,82].

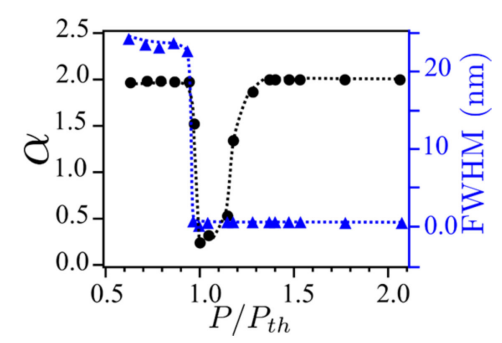

Figure 6. Lévy index $\alpha$ (circles) and FWHM (triangles) as a function of the normalized input pump power $P / P_{t h}$. Three statistical regimes of intensity fluctuations are observed: prelasing Gaussian with amplified spontaneous emission $(\alpha=2)$, Lévy-type RL $(0<\alpha<2)$ around the threshold, and Gaussian RL $(\alpha=2)$ well above the threshold. The sharp decrease in $\alpha$ at the first Gaussian-to-Lévy transition nicely coincides with the abrupt change in FWHM at the RL threshold. (Reproduced with permission from ref. [68]).

We further notice in Figure 6 that the abrupt decrease in the Lévy index $\alpha$ at the onset of RL behavior is closely related to the sharp linewidth reduction observed in Figure 2. Therefore, our results for the Er-RFL system corroborate the suggestion of [93], that the transition from the Gaussian to the Lévy regime in RLs could be used as a universal identifier of the RL threshold (however, see also the comment below on the results of ref. [97]). In fact, we also included in Figure 6 the FWHM measurement, whose drastic change at the RL threshold nicely coincides with the first Gaussian-to-Lévy statistical transition. For higher values of the input power, after reaching a minimum around the threshold, the index $\alpha$ smoothly rises back to the Gaussian value $\alpha=2$ achieved above the threshold.

We finally discuss the connection of the above findings with the photonic spin-glass behavior recently reported in the Er-RFL system [68,69].

As mentioned in Section 3.2, the characterization of the photonic RSB glassy phase in the RL regime requires the calculation of a specific two-point correlation function, which in the present context, can be defined either among the mode amplitudes, mode phases, or intensity fluctuations. In the latter case, that can be accessed experimentally, where the replica overlap parameter is defined as [42,79]:

$$
q_{\gamma \beta}=\frac{\sum_{k} \Delta_{\gamma}(k) \Delta_{\beta}(k)}{\sqrt{\left[\sum_{k} \Delta_{\gamma}^{2}(k)\right]\left[\sum_{k} \Delta_{\beta}^{2}(k)\right]}},
$$

where $\gamma, \beta=1,2, \ldots, N_{s}$ denote the replica labels $\left(N_{s}=5000\right.$ in ref. [68]), the average intensity at the wavelength indexed by $k$ reads $\left\langle I>(k)=\sum_{\gamma=1}^{N_{s}} I_{\gamma}(k) / N_{s}\right.$, and the intensity fluctuation is 
$\Delta_{\gamma}(k)=I_{\gamma}(k)-<I>(k)$. In the photonic context, each output spectrum is considered a replica, i.e., a copy of the Er-RFL system under fairly identical experimental conditions. The PDF P $(q)$ represents the distribution of values $q=q_{\gamma \beta}$ of the mode-mode correlations between intensity fluctuations, Equation (8). In fact, as discussed in the previous subsection, $\mathrm{P}(q)$ is analogous to the Parisi order parameter in the RSB spin-glass theory of disordered magnetic systems [84]. In the present context, the distribution $\mathrm{P}(q)$ signalizes a photonic replica-symmetric paramagnetic prelasing regime if its maximum occurs exclusively at $q_{\max }=0$ (no RSB), or a RSB spin-glass RL phase if the maximum also assumes values $\left|q_{\max }\right| \neq 0$ (RSB) (see Section 3.2).

Figure 7 shows a remarkable agreement between the onset of the Lévy statistical regime of intensity fluctuations and the emergence of the RSB glassy RL phase in Er-RFL. Indeed, we observe that both the Lévy and spin-glass behaviors are simultaneously present around the RL threshold. Therefore, besides signaling the Gaussian-to-Lévy shift in the statistical characteristics of intensity fluctuations, which is illustrated by Figure 6, the RL threshold also marks the sharp phase transition from the $q_{\max } \cong 0$ replica-symmetric paramagnetic prelasing regime with amplified spontaneous emission to the $q_{\max } \cong 1$ spin-glass RL phase with RSB properties. This coinciding behavior, firstly demonstrated in a 3D RL [82], is thus also shared by the Er-RFL system, although a recent report [97] has pointed out that this might not be a universal property of RL systems. Indeed, in ref. [97] it was demonstrated that a rigorous connection between the photonic phases and the statistics of intensity fluctuations is not mandatory, so that there can be circumstances in which, for example, a glassy phase emerges along with a Gaussian statistical regime of fluctuations. Therefore, though a complete theoretical understanding of such a finding is still lacking, it is possible in some instances to trace back the common physical origin of the Lévy and glassy behaviors to the Langevin equations for the amplitudes of the normal modes, which, as discussed, are the basis on which to explain both the statistical regimes of intensity fluctuations and the photonic RSB spin-glass behavior of RL systems.

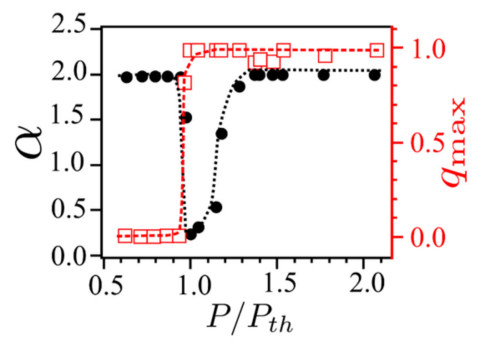

Figure 7. Lévy index $\alpha$ (circles) and overlap parameter $|q|=q_{\max }$ at which the PDF $\mathrm{P}(q)$ of the photonic replica overlaps with output intensity fluctuations at a maximum (squares) as a function of the normalized input power $P / P_{t h}$. The abrupt decrease in the parameter $\alpha$ at the first Gaussian-to-Lévy transition coincides nicely with the photonic phase transition observed from the $q_{\max } \cong 0$ replica-symmetric paramagnetic prelasing behavior, with amplified spontaneous emission, to the $q_{\max } \cong 1$ replica-symmetry-breaking (RSB) spin-glass phase of the Er-RFL system. Dotted lines are a guide to the eyes. (Reproduced with permission from ref. [68].)

\section{Summary and Discussion}

In this work, we described the operation of an RFL based on an erbium-doped fiber imprinted with randomly-spaced Bragg gratings. By exciting the fiber with a CW diode laser, we investigated the statistical fluctuations of the output intensity emitted by the erbium ions in the near infrared. The results allowed us to identify different statistical regimes for excitation powers $P$ below, around, and above the laser threshold, $P_{t h}$. In particular, the Lévy statistics were clearly identified for $P \approx P_{t h}$, while the Gaussian statistics were observed for $P<P_{t h}$ and $P>P_{t h}$. Moreover, we also found that the probability distribution for the emitted intensity around the laser threshold reveals a glassy phase of light that is compatible with an RSB analogue of the spin-glass phase transition. 
The studies reported here led to a deeper understanding of the physical processes underlying the RFL operation, and the results were also consistent with recent findings in $2 \mathrm{D}$ and 3D RLs.

As illustrated by the references cited in this work, the research on RFLs is still a hot subject after ten years since the first demonstration. The low fabrication cost, small fiber length, and simple operation scheme enable various potential applications of RFLs, for example, in imaging, sensing, and optofluidics. Further research with a basis on the investigation of the intensity fluctuations in RFL systems may include the study of their temporal dynamics, as well as the emergency of extreme events, analogues of rogue waves, and photonic turbulent transitions around the excitation threshold.

Acknowledgments: We thank S.J.M. Carreño, S.I. Fewo, M. Gagné, V. Jerez, R. Kashyap, B.C. Lima, L.J.Q. Maia, A.L. Moura, P.I.R. Pincheira and A.F. Silva for the fruitful collaboration. We also acknowledge the financial support from the Brazilian Agencies: Conselho Nacional de Desenvolvimento Científico e Tecnológico (CNPq) and Fundação de Amparo à Ciência e Tecnologia do Estado de Pernambuco (FACEPE). The work was performed in the framework of the National Institute of Photonics (INCT de Fotônica) and PRONEX-CNPq/FACEPE projects.

Author Contributions: C.B.d.A., A.S.L.G. and E.P.R. contributed equally to this review.

Conflicts of Interest: The authors declare no conflict of interest.

\section{References}

1. Ambartsumyan, R.V.; Basov, N.G.; Kryukov, P.G.; Letokhov, V.S. Laser with nonresonant feedback. JETP Lett. 1966, 3, 167-169.

2. Letokhov, V.S. Generation of light by a scattering medium with negative resonance absorption. Sov. Phys. JETP 1968, 26, 835-840.

3. Ambartsumyan, R.V.; Kryukov, P.G.; Letokhov, V.S. Dynamics of emission line narrowing for a laser with nonresonant feedback. Sov. Phys. JETP 1967, 24, 1129-1134.

4. Ambartsumyan, R.V.; Basov, N.G.; Letokhov, V.S. Frequency stability of a HeNe laser with nonresonant feedback. IEEE Trans. Instrum. Meas. 1968, 17, 338-343.

5. Ambartsumyan, R.V.; Kryukov, P.G.; Letokhov, V.S.; Matveets, Y.A. Emission statistics of a laser with nonresonant feedback. JETP Lett. 1967, 5, 312-314.

6. Letokhov, V.S. Stimulated radio emission of the interstellar medium. JETP Lett. 1966, 4, 321-323.

7. Lavrinovich, N.N.; Letokhov, V.S. The possibility of laser effect in stellar atmospheres. Sov. Phys. JETP 1975, 40, 800-805.

8. Letokhov, V.S. Astrophysical lasers. Quantum Electron. 2002, 32, 1065-1079. [CrossRef]

9. Johansson, S.; Letokhov, V.S. Astrophysical lasers operating in optical Fe II lines in stellar ejecta of Eta Carinae. Astron. Astrophys. 2004, 428, 427-509. [CrossRef]

10. Noginov, M.A. Solid State Random Lasers; Springer Series in Optical Sciences; Springer: New York, NY, USA, 2005.

11. Lawandy, N.M.; Balachandran, R.M.; Gomes, A.S.L.; Sauvian, E. Laser action in strongly scattering medium. Nature 1994, 368, 436-438. [CrossRef]

12. Ye, L.; Zhao, C.; Feng, Y.; Gu, B.; Cui, Y.; Lu, Y. Study on the polarization of random lasers from dye-doped nematic liquid crystals. Nanoscale Res. Lett. 2017, 12, 27. [CrossRef] [PubMed]

13. Abegão, L.M.G.; Manoel, D.S.; Otuka, A.J.G.; Ferreira, P.H.D.; Vollet, D.R.; Donatti, D.A.; De Boni, L.; Mendonça, C.R.; de Vicente, F.S.; Rodrigues, J.J., Jr.; et al. Random laser emission from a rhodamine B-doped GPTS/TEOS-derived organic/sílica monolithic xerogel. Laser Phys. Lett. 2017, 14, 065801. [CrossRef]

14. Shasti, M.; Coutino, P.; Mukherjee, S.; Varanytsia, A.; Smith, T.; Luchette, A.P.; Sukhomlinova, L.; Kosa, T.; Munoz, A.; Taheri, B. Reverse mode switching of the random laser emission in dye doped liquid crystals under homogeneous and inhomogeneous electric fields. Photonics Res. 2016, 4, 7-12. [CrossRef]

15. Anderson, B.R.; Gunawidjaja, R.; Eilers, H. Self-healing organic-dye-based random lasers. Opt. Lett. 2015, 40, 577-580. [CrossRef] [PubMed]

16. Gomes, A.S.L.; Carvalho, M.T.; Dominguez, C.T.; de Araújo, C.B.; Prasad, P.N. Direct three-photon excitation of upconversion random laser emission in a weakly scattering organic colloidal system. Opt. Express 2014, 22, 14305-14310. [CrossRef] [PubMed] 
17. Knitter, S.; Kues, M.; Fallnich, C. Emission polarization of random lasers in organic dye solutions. Opt. Lett. 2012, 37, 3621-3623. [CrossRef] [PubMed]

18. Chen, Y.; Herrnsdorf, J.; Guilhabert, B.; Zhang, Y.; Watson, I.M.; Gu, E.; Laurand, N.; Dawson, M.D. Colloidal quantum dot random laser. Opt. Express 2011, 19, 2996-3003. [CrossRef] [PubMed]

19. Dominguez, C.T.; Gomes, M.A.; Macedo, Z.S.; de Araújo, C.B.; Gomes, A.S.L. Multi-photon excited coherent random laser emission in ZnO powders. Nanoscale 2015, 7, 317-323. [CrossRef] [PubMed]

20. Moura, A.L.; Jerez, V.; Maia, L.J.Q.; Gomes, A.S.L.; de Araújo, C.B. Multi-wavelength emission through self-induced second-order wave-mixing processes from a $\mathrm{Nd}^{3+}$ doped crystalline powder random laser. Sci. Rep. 2015, 5, 13816. [CrossRef] [PubMed]

21. Moura, A.L.; Carreño, S.J.M.; Pincheira, P.I.R.; Fabris, Z.V.; Maia, L.J.Q.; Gomes, A.S.L.; de Araújo, C.B. Tunable ultraviolet and blue light generation from $\mathrm{Nd}$ :YAB random laser bolstered by second-order nonlinear processes. Sci. Rep. 2016, 6, 27107. [CrossRef] [PubMed]

22. Polson, R.C.; Chipouline, A.; Vardeny, Z.V. Random lasing in $\pi$-conjugated films and infiltrated opals. Adv. Mater. 2001, 13, 760-764. [CrossRef]

23. Meng, X.; Fujita, K.; Murai, S.; Tanaka, K. Coherent random lasers in weakly scattering polymer films containing silver nanoparticles. Phys. Rev. A 2009, 79, 053817. [CrossRef]

24. Costela, A.; Garcia-Moreno, I.; Cerdan, L.; Martin, V.; Garcia, O.; Sastre, R. Dye-doped POSS solutions: Random nanomaterials for laser emission. Adv. Mater. 2009, 21, 4163-4166. [CrossRef]

25. Tulek, A.; Polson, R.C.; Vardeny, Z.V. Naturally occurring resonators in random lasing of $\pi$-conjugated polymer films. Nat. Phys. 2010, 6, 303-310. [CrossRef]

26. Polson, R.C.; Raikh, M.E.; Vardeny, Z.V. Random lasing from weakly scattering media; Spectrum universality in DOO-PPV polymer films. Phys. E 2002, 13, 1240-1242. [CrossRef]

27. Dos Santos, M.V.; Dominguez, C.T.; Schiavon, J.V.; Barud, H.S.; de Melo, L.S.A.; Ribeiro, S.J.L.; Gomes, A.S.L.; de Araújo, C.B. Random laser action from flexible biocellulose-based device. J. Appl. Phys. 2014, 115, 083108. [CrossRef]

28. Yu, S.F.; Yuen, C.; Lau, S.P.; Park, W.I.; Yi, G.-C. Random laser action in ZnO nanorod arrays embedded in ZnO epilayers. Appl. Phys. Lett. 2004, 84, 3241-3243. [CrossRef]

29. Wang, Z.; Shi, X.; Wei, S.; Sun, Y.; Wang, Y.; Zhou, J.; Shi, J.; Liu, D. Two-threshold silver nanowire-based random laser with different dye concentrations. Laser Phys. Lett. 2014, 11, 095002. [CrossRef]

30. Gao, F.; Morshed, M.M.; Bashar, S.B.; Zheng, Y.; Shi, Y.; Liu, J. Electrically pumped random lasing based on an Au-ZnO nanowire Schottky junction. Nanoscale 2015, 7, 9505-9509. [CrossRef] [PubMed]

31. Bashar, S.B.; Suja, M.; Morshed, M.; Gao, F.; Liu, J. An Sb-doped p-type ZnO nanowire based random laser diode. Nanotechnology 2016, 27, 065204. [CrossRef] [PubMed]

32. Baudouin, Q.; Mercadier, N.; Guarrera, V.; Guerin, W.; Kaiser, R. A cold-atom random laser. Nat. Phys. 2013, 9, 357-360. [CrossRef]

33. Van der Molen, K.L.; Mosk, A.P.; Lagendijk, A.D. Intrinsic intensity fluctuations in random lasers. Phys. Rev. A 2006, 74, 053808. [CrossRef]

34. Van der Molen, K.L.; Tjerkstra, R.W.; Mosk, A.P.; Lagendijk, A.D. Spatial extent of random laser modes. Phys. Rev. Lett. 2007, 98, 143901. [CrossRef] [PubMed]

35. Wiersma, D.S. The physics and applications of random lasers. Nat. Phys. 2008, 4, 359-367. [CrossRef]

36. Leonetti, M.; Conti, C.; Lopez, C. The mode-locking transition of random lasers. Nat. Photonics 2011, 5, 615-617. [CrossRef]

37. Ignesti, E.; Tommasi, F.; Fini, L.; Martelli, F.; Azzali, N.; Cavalieri, S. A new class of optical sensors: A random laser based device. Sci. Rep. 2016, 6, 35225. [CrossRef] [PubMed]

38. Bhaktha, B.N.S.; Bachelard, N.; Noblin, X.; Sebbah, P. Optofluidic random laser. Appl. Phys. Lett. 2012, 101, 151101. [CrossRef]

39. Bachelard, N.; Gigan, S.; Noblin, X.; Sebbah, P. Adaptive pumping for spectral control of random lasers. Nat. Phys. 2014, 10, 426-431. [CrossRef]

40. Redding, B.; Choma, M.A.; Cao, H. Speckle-free laser imaging using random laser illumination. Nat. Photonics 2012, 6, 355-359. [CrossRef] [PubMed]

41. Luan, F.; Gu, B.B.; Gomes, A.S.L.; Yong, K.T.; Wen, S.C.; Prasad, P.N. Lasing in nanocomposite random media. Nano Today 2015, 10, 168-192. [CrossRef] 
42. Ghofraniha, N.; Viola, I.; Di Maria, F.; Barbarella, G.; Gigli, G.; Leuzzi, L.; Conti, C. Experimental evidence of replica symmetry breaking in random lasers. Nat. Commun. 2015, 6, 6058. [CrossRef] [PubMed]

43. De Mattos, C.J.S.; Menezes, L.S.; Brito-Silva, A.M.; Gámez, M.A.M.; Gomes, A.S.L.; de Araújo, C.B. Random fiber laser. Phys. Rev. Lett. 2007, 99, 153903. [CrossRef] [PubMed]

44. Lizárraga, N.; Puente, N.P.; Chaikina, E.I.; Leskova, T.A.; Méndez, E.R. Single-mode Er-doped fiber random laser with distributed Bragg grating feedback. Opt. Express 2009, 17, 395-404. [CrossRef] [PubMed]

45. Gagné, M.; Kashyap, R. Demonstration of a $3 \mathrm{~mW}$ threshold Er-doped random fiber laser based on a unique fiber Bragg grating. Opt. Express 2009, 17, 19067-19074. [CrossRef] [PubMed]

46. Turitsyn, S.K.; Babin, S.A.; El-Taher, A.E.; Harper, P.; Churkin, D.V.; Kablukov, S.I.; Ania-Castanon, J.D.; Karalekas, V.; Podivilov, E.V. Random distributed feedback fibre laser. Nat. Photonics 2010, 4, 231-235. [CrossRef]

47. Turitsyn, S.K.; Babin, S.A.; Churkin, D.V.; Vatnik, I.D.; Nikulin, M.; Podivilov, E.V. Random distributed feedback fibre lasers. Phys. Rep. 2014, 542, 133-193. [CrossRef]

48. Churkin, D.V.; Sugavanam, S.; Vatnik, I.D.; Wang, Z.; Podivilov, E.V.; Babin, S.A.; Rao, Y.; Turitsyn, S.K. Recent advances in fundamentals and applications of random fiber lasers. Adv. Opt. Photonics 2015, 7, 516-569. [CrossRef]

49. Wang, Z.; Wu, H.; Fan, M.; Zhang, L.; Rao, Y.; Zhang, W.; Jia, X. High power random fiber laser with short cavity length: Theoretical and experimental investigations. IEEE J. Sel. Top. Quantum Electron. 2015, 21, 0900506.

50. Li, S.W.; Ma, R.; Rao, Y.J.; Zhu, Y.Y.; Wang, Z.N.; Jia, X.H.; Li, J. Random distributed feedback fiber laser based on combination of Er-doped fiber and single-mode fiber. IEEE J. Sel. Top. Quantum Electron. 2015, 21, 0900406.

51. Wu, H.; Wang, Z.; Fan, M.; Zhang, L.; Zhang, W.; Ra, Y. Role of the mirror's reflectivity in forward-pumped random fiber laser. Opt. Express 2015, 23, 1421-1427. [CrossRef] [PubMed]

52. Zhang, H.; Zhou, P.; Wang, X.; Du, X.; Xiao, H.; Xu, X. Hundred-watt-level high power random distributed feedback Raman fiber laser at $1150 \mathrm{~nm}$ and its application in mid-infrared laser generation. Opt. Express 2015, 23, 17138-17144. [CrossRef] [PubMed]

53. Zhang, W.L.; Ma, R.; Tang, C.H.; Rao, Y.J.; Zeng, X.P.; Yang, Z.J.; Wang, Z.N.; Gong, Y.; Wang, Y.S. All optical mode controllable Er-doped random fiber laser with distributed Bragg gratings. Opt. Lett. 2015, 40, 3181-3184. [CrossRef] [PubMed]

54. Wang, L.; Dong, X.; Shum, P.P.; Liu, X.; Su, H. Random laser with multiphase-shifted Bragg grating in Er/Yb-codoped fiber. J. Lightwave Technol. 2015, 33, 95-99. [CrossRef]

55. Du, X.; Zhang, H.; Wang, X.; Zhou, P.; Liu, Z. Investigation on random distributed feedback Raman fiber laser with linear polarized output. Photonics Res. 2015, 3, 28-31. [CrossRef]

56. Tang, Y.; Xu, J. A random Q-switched fiber laser. Sci. Rep. 2015, 5, 9338. [CrossRef] [PubMed]

57. Yao, B.C.; Rao, Y.J.; Wang, Z.N.; Wu, Y.; Zhou, J.H.; Wu, H.; Fan, M.Q.; Cao, X.L.; Zhang, W.L.; Chen, Y.F.; et al. Graphene based widely-tunable and singly-polarized pulse generation with random fiber lasers. Sci. Rep. 2015, 5, 18526. [CrossRef] [PubMed]

58. Zhang, L.; Jiang, H.; Yang, X.; Pan, W.; Feng, Y. Ultra-wide wavelength tuning of a cascaded Raman random fiber laser. Opt. Lett. 2016, 41, 215-218. [CrossRef] [PubMed]

59. Du, X.; Zhang, H.; Wang, X.; Zhou, P.; Liu, Z. Short cavity-length random fiber laser with record power and ultrahigh efficiency. Opt. Lett. 2016, 41, 571-574. [CrossRef] [PubMed]

60. Dontsova, E.I.; Kablukov, S.I.; Vatnik, I.D.; Babin, S.A. Frequency doubling of Raman fiber lasers with random distributed feedback. Opt. Lett. 2016, 41, 1439-1442. [CrossRef] [PubMed]

61. Hu, Z.; Liang, Y.; Qian, X.; Gao, P.; Xie, K.; Jiang, H. Polarized random laser emission from an oriented disorder polymer optical fiber. Opt. Lett. 2016, 41, 2584-2587. [CrossRef] [PubMed]

62. Ardakani, A.G.; Rafieipour, P. Investigation of one-dimensional Raman random lasers based on the finite-difference-time-domain method: Presence of mode competition and higher-order Stokes and anti-Stokes modes. Phys. Rev. A 2016, 93, 023833. [CrossRef]

63. Zhang, W.L.; Song, Y.B.; Zeng, X.P.; Ma, R.; Yang, Z.J.; Rao, Y.J. Temperature-controlled mode selection of Er-doped random fiber laser with disordered Bragg gratings. Photonics Res. 2016, 4, 102-105. [CrossRef] 
64. Wu, H.; Wang, Z.; Rao, Y. Tailoring the properties of cw random fiber lasers. In Proceedings of the Advanced Photonics Congress 2016 (IPR, NOMA, Sensors, Networks, SPPCom, SOF), Vancouver, NA, Canada, 18-20 July 2016.

65. Babin, S.A.; Zlobina, E.A.; Kablukov, S.I.; Podivilov, E.V. High-order random Raman lasing in a PM fiber with ultimate e efficiency and narrow bandwidth. Sci. Rep. 2016, 6, 22625. [CrossRef] [PubMed]

66. Zhang, W.L.; Zheng, M.Y.; Ma, R.; Gong, C.Y.; Yang, Z.J.; Peng, G.D.; Rao, Y.J. Fiber-type random laser based on a cylindrical waveguide with a disordered cladding layer. Sci. Rep. 2016, 6, 26473. [CrossRef] [PubMed]

67. González, I.R.R.; Lima, B.C.; Pincheira, P.I.R.; Brum, A.A.; Macêdo, A.M.S.; Vasconcelos, G.L.; Menezes, L.S.; Raposo, E.P.; Gomes, A.S.L.; Kashyap, R. Turbulence hierarchy in a random fibre laser. Nat. Commun. 2017, 8, 15731. [CrossRef] [PubMed]

68. Lima, B.C.; Gomes, A.S.L.; Pincheira, P.I.R.; Moura, A.L.; Gagné, M.; Raposo, E.P.; de Araújo, C.B.; Kashyap, R. Observation of Lévy statistics in one-dimensional erbium-based random fiber laser. J. Opt. Soc. Am. B 2017, 34, 293-299. [CrossRef]

69. Gomes, A.S.L.; Lima, B.C.; Pincheira, P.I.R.; Moura, A.L.; Gagné, M.; Raposo, E.P.; de Araújo, C.B.; Kashyap, R. Glassy behavior in a one-dimensional continuous-wave erbium-doped random fiber laser. Phys. Rev. A 2016, 94, 011801. [CrossRef]

70. Hokr, B.H.; Cerjan, A.; Thompson, J.V.; Yuan, L.; Liew, S.F.; Bixler, J.N.; Noojin, G.D.; Thomas, R.J.; Cao, H.; Stone, A.D.; et al. Evidence of Anderson localization effects in random Raman lasing. SPIE Proc. 2016, 9731, 973110.

71. Goodman, J.W. Speckle Phenomena in Optics: Theory and Applications; Roberts \& Company: Englewood, CO, USA, 2007.

72. Zhu, G.; Gu, L.; Noginov, M.A. Experimental study of instability in a random laser with immobile scatterers. Phys. Rev. A 2012, 85, 043801. [CrossRef]

73. Angelani, L.; Conti, C.; Ruocco, G.; Zamponi, F. Glassy behavior of light in random lasers. Phys. Rev. B 2006, 74, 104207. [CrossRef]

74. Angelani, L.; Conti, C.; Ruocco, G.; Zamponi, F. Glassy behavior of light. Phys. Rev. Lett. 2006, 96, 065702. [CrossRef] [PubMed]

75. Leuzzi, L.; Conti, C.; Folli, V.; Angelani, L.; Ruocco, G. Phase diagram and complexity of mode-locked lasers: From order to disorder. Phys. Rev. Lett. 2009, 102, 083901. [CrossRef] [PubMed]

76. Conti, C.; Leuzzi, L. Complexity of waves in nonlinear disordered media. Phys. Rev. B 2011, 83, 134204. [CrossRef]

77. Antenucci, F.; Conti, C.; Crisanti, A.; Leuzzi, L. General phase diagram of multimodal ordered and disordered lasers in closed and open cavities. Phys. Rev. Lett. 2015, 114, 043901. [CrossRef] [PubMed]

78. Antenucci, F.; Crisanti, A.; Leuzzi, L. Complex spherical $2+4$ spin glass: A model for nonlinear optics in random media. Phys. Rev. A 2015, 91, 053816. [CrossRef]

79. Antenucci, F.; Crisanti, A.; Leuzzi, L. The glassy random laser: Replica symmetry breaking in the intensity fluctuations of emission spectra. Sci. Rep. 2015, 5, 16792. [CrossRef] [PubMed]

80. Antenucci, F.; Crisanti, A.; Ibáñez-Berganza, M.; Marruzzo, A.; Leuzzi, L. Statistical mechanics models for multimode lasers and random lasers. Philos. Mag. 2016, 96, 704-731. [CrossRef]

81. Raposo, E.P.; Gomes, A.S.L. Analytical solution for the Lévy-like steady-state distribution of intensities in random lasers. Phys. Rev. A 2015, 91, 043827. [CrossRef]

82. Gomes, A.S.L.; Raposo, E.P.; Moura, A.L.; Fewo, S.I.; Pincheira, P.I.R.; Jerez, V.; Maia, L.J.Q.; de Araújo, C.B. Observation of Lévy distribution and replica symmetry breaking in random lasers from a single set of measurements. Sci. Rep. 2016, 6, 27987. [CrossRef] [PubMed]

83. Crisanti, A.; Sommers, H.-J. The spherical p-spin interaction spin glass model: The statics. Z. Phys. B 1992, 87, 341-354. [CrossRef]

84. Mézard, M.; Parisi, G.; Virasoro, M.A. Spin Glass Theory and Beyond; World Scientific: Singapore, 1987.

85. Pincheira, P.I.R.; Silva, A.F.; Carreño, S.J.M.; Moura, A.L.; Fewo, S.I.; Raposo, E.P.; Gomes, A.S.L.; de Araújo, C.B. Observation of photonic to paramagnetic spin-glass transition in specially-designed $\mathrm{TiO}_{2}$ particles-based dye-colloidal random laser. Opt. Lett. 2016, 41, 3459-3462. [CrossRef] [PubMed]

86. O’Bryan, I.C.L.; Sargent, I.M. Theory of multimode laser operation. Phys. Rev. A 1973, 8, 3071-3092. [CrossRef] 
87. Schenzle, A.; Brand, H. Multiplicative stochastic processes in statistical physics. Phys. Rev. A 1979, 20, 1628-1647. [CrossRef]

88. Samorodnitsky, G.; Taqqu, M.S. Stable Non-Gaussian Random Processes; Chapman and Hall: London, UK, 1994.

89. Uppu, R.; Mujumdar, S. Exponentially tempered Lévy sums in random lasers. Phys. Rev. Lett. 2015, 114, 183903. [CrossRef]

90. Mantegna, R.N.; Stanley, H.E. Stochastic process with ultraslow convergence to a Gaussian: The truncated Lévy flight. Phys. Rev. Lett. 1994, 73, 2946-2949. [CrossRef] [PubMed]

91. Bartumeus, F.; Raposo, E.P.; Viswanathan, G.M.; da Luz, M.G.E. Stochastic optimal foraging: Tuning intensive and extensive dynamics in random searches. PLoS ONE 2014, 9, e106373. [CrossRef] [PubMed]

92. Lepri, S.; Cavalieri, S.; Oppo, G.-L.; Wiersma, D.S. Statistical regimes of random laser fluctuations. Phys. Rev. A 2007, 75, 063820. [CrossRef]

93. Uppu, R.; Mujumdar, S. Lévy exponents as universal identifiers of threshold and criticality in random lasers. Phys. Rev. A 2014, 90, 025801. [CrossRef]

94. McCulloch, J.H. Simple consistent estimators of stable distribution parameters. Commun. Stat. Simul. 1986, 15, 1109-1136. [CrossRef]

95. Uppu, R.; Tiwari, A.K.; Mujumdar, S. Identification of statistical regimes and crossovers in coherent random laser emission. Opt. Lett. 2012, 37, 662-664. [CrossRef] [PubMed]

96. Uppu, R.; Mujumdar, S. Dependence of the Gaussian-Lévy transition on the disorder strength in random lasers. Phys. Rev. A 2013, 87, 013822. [CrossRef]

97. Tommasi, F.; Ignesti, E.; Lepri, S.; Cavalieri, S. Robustness of replica symmetry breaking phenomenology in random laser. Sci. Rep. 2016, 6, 37113. [CrossRef] [PubMed]

(C) 2017 by the authors. Licensee MDPI, Basel, Switzerland. This article is an open access article distributed under the terms and conditions of the Creative Commons Attribution (CC BY) license (http:/ / creativecommons.org/licenses/by/4.0/). 